\title{
Estimation of Genetic Parameters for Perinatal Sucking Behavior of Italian Brown Swiss Calves
}

\author{
C. Maltecca, ${ }^{*}$ A. Rossoni,†‡ C. Nicoletti,† E. Santus,† K. A. Weigel, ${ }^{*}$ and A. Bagnatoł ${ }^{1}$ \\ *Dairy Science Department, University of Wisconsin, Madison 53706 \\ †Italian Brown Breeders Association, Loc. Ferlina 204, Bussolengo 37012, Italy \\ ‡Department of Veterinary Science and Technology for Food Safety, Università degli Studi di Milano, Via Celoria 10, 20133 Milano, Italy
}

\section{ABSTRACT}

Brown Swiss breeders sometimes experience difficulties in feeding calves because of the weak sucking ability of the calves in the early days of life. For the welfare of the calves, they should be suckled by their dams or should aggressively ingest colostrum immediately after birth. The composition of colostrum changes rapidly during the first few days of lactation, and the ability of calves to absorb the Ig decreases quickly as well. The aim of this study was to increase our knowledge of environmental and genetic components affecting the sucking response, to evaluate the possibility of selecting for this trait. Sucking ability was recorded in 3 categories (drank from the milk bucket nipple or bottle without help, drank with help, did not drink) at 5 postnatal meals $(6,12,24,48$, and $72 \mathrm{~h}$ from birth). Records were analyzed with 2 different models: a single-trait threshold sire model, in which all observations were analyzed as a single trait with 5 levels, and a multipletrait threshold liability sire model, in which meal-bymeal observations were analyzed as 5 different binary traits. Management procedures, the interval between birth and meals, parity, and season of birth were environmental factors affecting the variability in sucking ability. The heritability estimate for the single-trait analysis was 0.14 , whereas heritabilities for the multiple-trait analysis were $0.26,0.22,0.210 .12$, and 0.13 for the first, second, third, fourth, and fifth meal, respectively. Estimated genetic correlations among traits were high (0.82 to 0.99 ). This study suggests the possibility of selection based on sucking ability. Future collection of larger data sets on the sucking response of calves in the first 2 meals after birth would increase the accuracy of genetic parameter estimates.

Key words: sucking ability, functional trait, Brown Swiss, dairy cattle

Received March 9, 2007.

Accepted June 25, 2007.

${ }^{1}$ Corresponding author: alessandro.bagnato@unimi.it

\section{INTRODUCTION}

Calves with suboptimal feed intake during the first days of life exhibit greater postnatal mortality, and this can adversely affect the profitability of dairy farms. In Danish Holstein cattle, Hansen et al. (2003) found that $2.7 \%$ of calves did not survive to 2 wk of age. In American Brown Swiss cattle, Erf et al. (1990) found even more disconcerting results, showing that $6.4 \%$ of calves died by $2 \mathrm{~d}$ after birth. In European Brown Swiss, newborn calves sometimes do not show sufficient sucking instinct, which can often be lethal. This lack of sucking aptitude can limit the diffusion of the breed in an intensive farming system (Bulot 2004; Santus, 2004; Zemp, 2004).

In ungulates, as in other mammals, contact with the mother represents a multisensorial stimulation with which the young animal comes into contact at birth (Nowak et al., 1997). In the modern dairy industry, calves are separated from their mothers immediately after calving. This practice could be causal in affecting calves' motivation and aptitude to suck aggressively, because calves reared separately from their mothers are often fed by buckets. Additionally, they can only suck on objects in the pen, or on pen mates, to satisfy their sucking motivation (de Passilè, 2001). The exact stimuli that influence the first sucking by calves are not known. Along with environmental factors, Schaal et al. (2003) identified in rabbits a pheromone, 2methylbut-2-enal, that elicits a complex behavioral sequence that culminates in the attachment of a pup to the mother's nipple. The pheromone 2-methylbut-2enal is also present in cow's milk, but with no additional knowledge of its effect on sucking behavior in cattle, assumptions about the effect of this pheromone in calves that are separated from their mothers immediately after birth are difficult to infer (Blass, 2003).

Passive immunity in newborn calves occurs through the absorption of Ig from colostrum shortly after birth (Bush and Staley, 1980), and low serum Ig concentrations are directly related to long-term calf performance (Wittum and Perino, 1995; Faber et al., 2005). The colos- 
trum composition begins to change soon after calving, particularly the Ig concentrations, which decrease and ultimately disappear after $3 \mathrm{~d}$ postpartum. The energy and protein content of colostrum (i.e., its nutritional components) also decrease significantly (Blum and Hammon, 2000). For the aforementioned reasons, along with the decreased ability of calves to absorb colostral Ig after birth (Stott et al., 1979; Staley and Bush, 1985), the presence of sucking aptitude is very important for the health and welfare of calves immediately after birth. The aim of this study was to investigate the effects of environmental and genetic factors and estimate variance components for different measures of sucking behavior in the Brown Swiss breed to evaluate the possibility of genetic improvement of this trait.

\section{MATERIALS AND METHODS}

\section{Data Collection}

Observations regarding the capacity of the newborn calf to drink from a nipple bucket or bottle were collected from January 1998 to March 2003 in the Italian Brown Swiss population. Calves were not allowed to nurse from their dams. A nipple bucket or calf bottle with colostrum was offered to each calf. The feeding system and the person recording the phenotype were consistent within herd and during the measurement. Behavior of the calf with respect to the action of sucking from an artificial teat (nipple bucket or bottle) was measured (hereafter referred to as sucking ability). Sucking ability was recorded by the farmer as a categorical trait during the first 5 postnatal meals, at $6,12,24,48$ and $72 \mathrm{~h}$ after birth (SA6, SA12, SA24, SA48, and SA72, respectively). Sucking ability for each calf at each meal was recorded on a scale ranging from 0 to 2 , with 0 representing a calf that consumed colostrum or milk without help (no farmer intervention after the beginning of sucking to the end of the meal), 1 representing a calf that consumed colostrum or milk with some farmer intervention (calf sucked only if the farmer placed the teat in the mouth of the newborn), and 2 representing a calf that did not consume milk or colostrum (the calf did not suck, or even completely refused the teat). A total of 5,913 observations were recorded on 1,274 calves on 263 farms. An aggregate measure (SA) was also considered, which combined the aforementioned measures (SA6 to SA72) into a unique trait (SA) with repeated measures. The scale of this last trait was the same as noted previously $(0,1,2)$. Participation in the project was voluntary, ensuring that the farmers involved were motivated to record the sucking ability at different meals on the form provided according to the category definition. To guarantee reliable data collection, each farmer was specifically trained by personnel
Table 1. Summary statistics of the edited data set

\begin{tabular}{lr}
\hline Statistic & Number \\
\hline Calves & 855 \\
Observations & 4,275 \\
Sires with data & 187 \\
Herd classes & 110 \\
Season classes & 2 (399 Jan. to Jun.; 456 Jul. to Dec.) \\
Parity of dam classes & 2 (229 primiparous; 626 multiparous) \\
Twin classes & 2 (817 single; 38 twin) \\
\hline
\end{tabular}

of the Italian Brown Swiss Association, and breed inspectors regularly visited farms and monitored data collection. Farmers were trained in using an explanatory form and in simulating the recording of measurements.

\section{Data Editing}

Herds with $<3$ observations, along with individuals with missing date of birth or identification of dam, were excluded from the analysis. Furthermore, only individuals with complete information on all 6 postnatal meals were considered in the study. After editing, 4,275 phenotypes on 855 calves, sired by 187 bulls, in 110 herds in 23 Italian provinces were available for the analysis. Pedigree information was provided by the Italian Brown Swiss Association database. A summary of the data set is provided in Table 1.

\section{Statistical Analysis of Environmental Effects}

Given the scarce literature regarding postnatal sucking behavior, an exploratory analysis to assess the significance of various environmental components was carried out. Analysis of variance was performed on Snell scores (Snell, 1964) of sucking ability as a single trait and as multiple traits. Briefly, Snell scores are used to transform categorical variables to conform to intervals of the normal distribution.

Explanatory variables included in the analysis were: herd, parity, year $\times$ season of calving interaction, meal number, and twin status. The analysis was carried out by using PROC GLM of SAS (SAS Institute, Cary, NC). Least squares means for the meal number were obtained for SA only.

\section{Multiple-Trait Threshold-Liability Sire Model.} To estimate genetic and environmental variance components, correlations, and heritabilities, 2 different approaches were taken. A 5-variate threshold-liability model was used in the first analysis, similar to the model proposed by Gianola (1982) and used by Heringstad et al. (2006) to analyze clinical mastitis and nonreturn rate in different time periods. The model is as follows: 
Table 2. Frequencies of the different categories for different meals (Snell scores in parentheses)

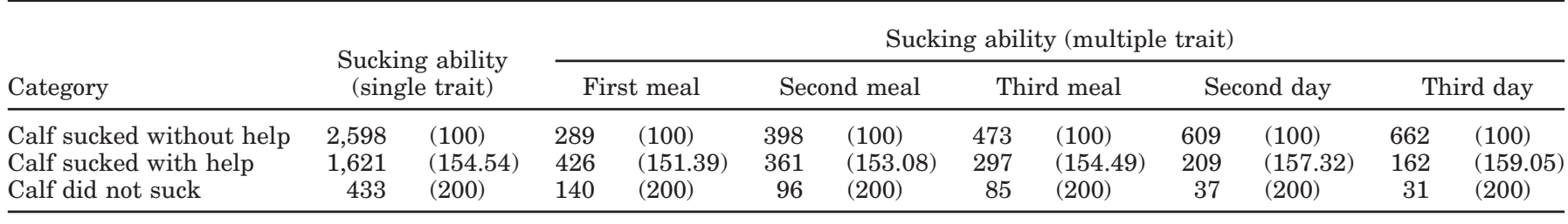

$$
\lambda=\boldsymbol{X} \beta+Z_{h} h+Z_{s} s+e,
$$

where $\lambda$ represents a vector of unobserved liabilities for the 5 meals, each representing a separate trait; $\boldsymbol{\beta}$ represents a vector of fixed effects, including year-season (12 levels) and parity of dam (2 levels, with 1 representing primiparous and 2 representing multiparous cows, respectively); $\boldsymbol{h}$ represents a vector of random herd effects (110 levels); $s$ represents a vector of sire transmitting abilities $(187 \times 5=935$ levels $)$; $\boldsymbol{e}$ is a vector of residuals; and $\boldsymbol{X}, \boldsymbol{Z}_{\boldsymbol{h}}$, and $\boldsymbol{Z}_{\boldsymbol{s}}$ represent corresponding incidence matrices. Residual variances were set equal to 1 . All residuals between individuals were considered independent but correlated within individuals, with a distribution of $\boldsymbol{e} \sim N\left(0, \boldsymbol{R}_{\mathbf{0}} \otimes I\right)$, where $\boldsymbol{R}_{\mathbf{0}}$ is a $5 \times 5$ covariance matrix with all diagonal elements set equal to 1. A Bayesian Markov chain Monte Carlo method (Sorensen and Gianola, 2002), as applied by Heringstad et al. (2006), was used to sample from the marginal posterior distributions. Independent uniform priors were assigned to the elements of $\beta$. Multivariate normal prior distributions were assigned to $\boldsymbol{h} \sim N\left(0, \boldsymbol{H}_{\mathbf{0}} \otimes I\right)$. In the same way, multivariate normal distribution priors were assigned to $\boldsymbol{s} \sim N\left(0, \boldsymbol{S}_{\mathbf{0}} \otimes \boldsymbol{A}\right)$, where $\boldsymbol{A}$ represents the numerator relationship matrix. Inverse Wishart prior distributions were used for the (co)variance matrices of herd $\left(\boldsymbol{H}_{\mathbf{0}}\right)$ and sire effects $\left(\boldsymbol{S}_{\mathbf{0}}\right)$, whereas off-diagonal elements of $\boldsymbol{R}_{\mathbf{0}}$ were assigned uniform priors bounded between -1 and 1 . Thresholds were assumed to be distributed as ordered statistics from a uniform distribution.

Single-Trait Threshold Sire Model. In the second approach, a univariate threshold model was used. The model was specified as follows:

$$
\lambda=X \beta+Z_{h} h+Z_{p} p+Z_{s} s+e
$$

where $\lambda$ represents a vector of liabilities for the 5 meals represented as a single trait with repeated observations; $\boldsymbol{p}$ represents a vector of permanent environmental effects, with $\boldsymbol{Z}_{\boldsymbol{p}}$ representing the corresponding incidence matrix; and all other factors in the model as described above. In this case, a Bayesian approach similar to the one outlined above was also used. In both approaches, visual inspections of trace plots were used to assess the number of iterations and burn-in length required, and a single chain of 100,000 samples with a burn-in of 10,000 samples was used for inferences.

\section{RESULTS AND DISCUSSION}

The distribution of sucking ability is shown in Table 2 . The largest proportion of animals sucked without assistance or with minor assistance, and the frequency of animals that did not suck at all decreased as calves were offered more meals. Twin calves did not differ from singletons in sucking ability at any time after birth. Herd effects were highly significant for all traits ( $P<0.01$, not shown), suggesting that management practices strongly affect the ability of a calf to learn or express the correct sucking behavior during the first days of life. Nevertheless, in this analysis herd represented the only management effect that could be taken into account. Admittedly, it is a broad environmental factor that clearly should be evaluated in more detail in future analyses of this trait. The trend of least squares means for the meal number factor for SA (highly significant at $P<0.01$ ), shown in Figure 1 , suggests that earlier measures of sucking ability are expected to better represent the innate nursing aptitude of the individual. With increasing time from birth, the calves learned to suck, and the number of individuals that refused to suck or consumed colostrum or milk with the farmer's help decreased substantially.

Figure 1 depicts how the sucking response was affected by herd management policies. Many factors related to herd management can affect the sucking ability of the newborn calf. Among these, the factors that probably have the largest effect are calf management practices, such as the feeding system used (Appleby and Weary, 2001), the handling of the calf (Mitchell et al., 1988), the type of housing (Lay et al., 1992), changes in housing conditions during the first few days (Friend et al., 1985, 1988), and the handling of the mother during the prenatal period (Lay et al., 1997). This study found that the proportion of calves experiencing difficulties decreased substantially after the first day of life (Figure 2). This could be attributed to an innate ability to survive, and hence a willingness to learn how to consume from an artificial teat, and to the farmer's help 


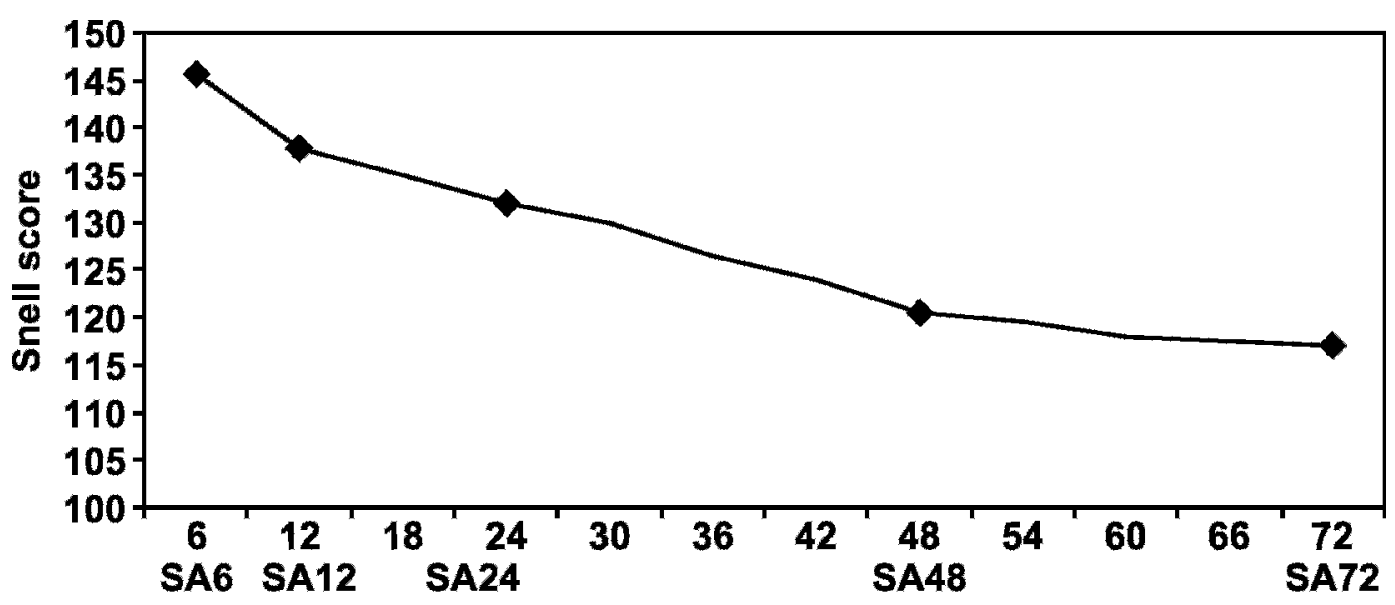

Birth to meal interval (h)

Figure 1. Least squares means for sucking ability at meals at different times from birth (SA6 = sucking ability at first meal; SA12 = sucking ability at second meal; SA24 = sucking ability at third meal; SA48 = sucking ability on second day; SA72 = sucking ability on third day).

during the first day of life. With respect to parity effects, the literature shows that primiparous cows exhibit more calving problems and a greater stillbirth rate compared with multiparous cows (Harbers et al., 2000; Johanson and Berger, 2003; Steinbock et al., 2003). In this study, calves from primiparous dams had a decreased sucking ability as compared with calves from multiparous dams ( $P<0.05$, data not shown). Calving difficulties might negatively affect the vitality of the calves and, indirectly, their sucking ability or vigor. Further studies involving data regarding calving ease and stillbirths, which were not available for this analysis, could shed light on this hypothesis. Season of birth also accounted for differences in sucking ability, with larger differences in earlier meals (Figure 2). Results showed that sucking ability was greater during the warm season. In fact, during winter, when temperatures were $-10^{\circ} \mathrm{C}$ and humidity increased, calves suffered more respiratory problems, which could have affected their strength and may have influenced their willingness to eat. This hypothesis is supported by the findings of previous studies showing an increase of $2 \%$ in the stillbirth rate during the winter (Harbers et al., 2000; Steinbock et al., 2003).

Figure 3 shows the posterior mean of heritability for the liability to deficiency of SA, which was estimated as $0.14( \pm 0.05)$. No previous analyses are available for comparison. An exploratory analysis performed on the

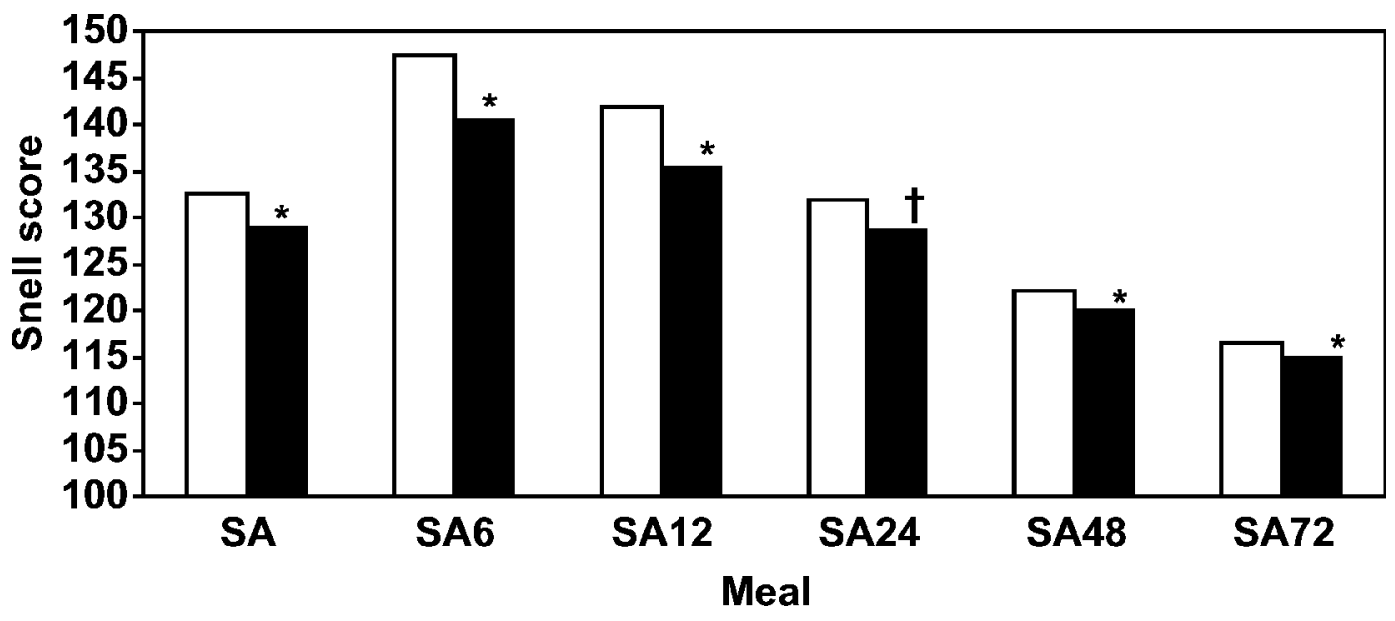

Figure 2. Least squares means for different seasons: $(\square)$ cold season, ( $\square$ ) hot season. Results of single-trait (SA) and multiple-trait (all others) analysis (SA = combined sucking ability over all meals; SA6 = sucking ability at first meal; SA12 = sucking ability at second meal; SA24 = sucking ability at third meal; SA48 = sucking ability on second day; SA72 = sucking ability on third day). $* P<0.05 ; \dagger P<0.10$. 


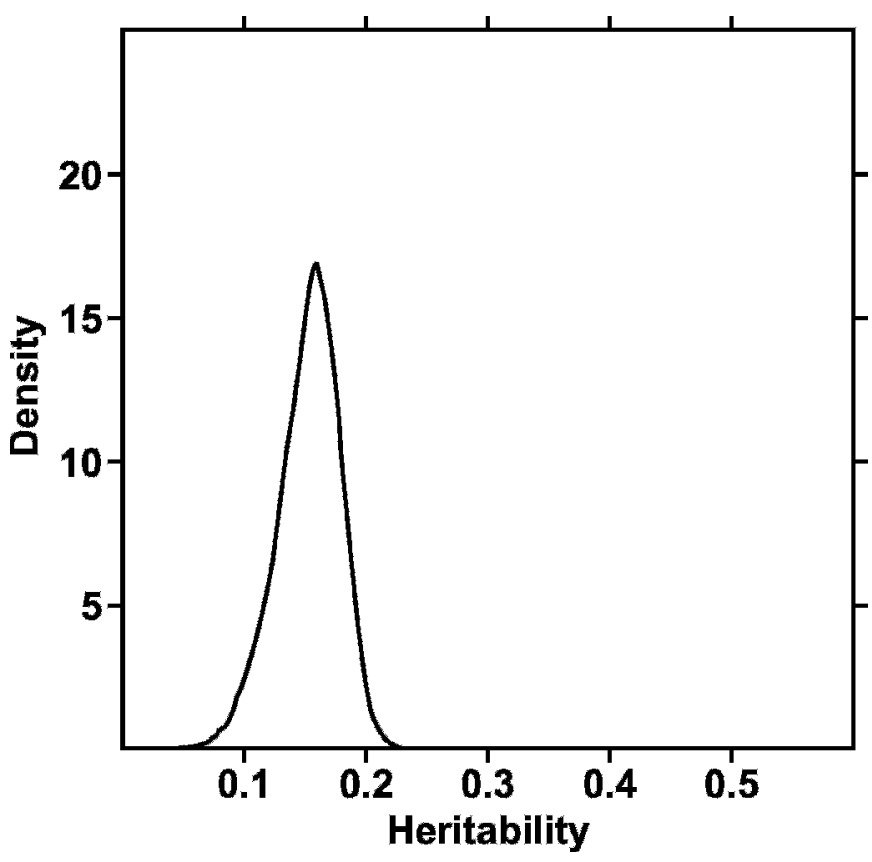

Figure 3. Posterior distribution of heritability $\left[\mathrm{h}^{2}=\right.$ $\left.4 \sigma_{\mathrm{s}}^{2} /\left(\sigma_{\mathrm{h}}^{2}+\sigma_{\mathrm{s}}^{2}+\sigma_{\mathrm{p}}^{2}+\sigma_{\mathrm{e}}^{2}\right)\right]$ of liability to sucking deficiency in the singletrait liability analysis.

same data set with the Snell scores of the variable SA and a linear model (data not shown) provided an estimated heritability of 0.08 . Posterior means of heritabilities for liability to deficiency of SA6, SA12, SA24, SA48, and SA72 are depicted in Figure 4. Estimates were $0.26( \pm 0.09), 0.23( \pm 0.08), 0.22( \pm 0.09), 0.13( \pm$ 0.08 ), and $0.13( \pm 0.07)$ for SA6, SA12, SA24, SA48, and SA72, respectively. The same analysis performed with Snell scores and a multiple-trait linear model produced estimates of roughly half those obtained with the threshold liability model. Table 3 provides estimates for genetic correlations (above the diagonal) and phenotypic correlations (below the diagonal) from the multivariate model. Genetic correlation estimates ranged from 0.84 to 0.99 , whereas phenotypic correlations ranged from 0.57 and 0.97 . Both were greatest for adja-

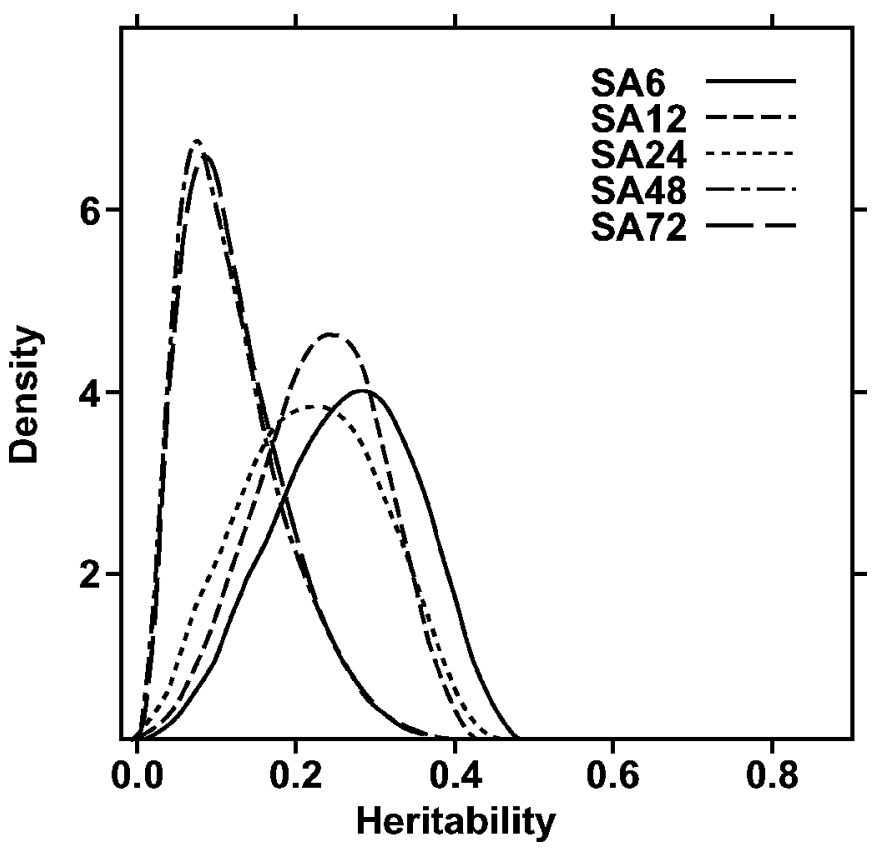

Figure 4. Posterior distributions of heritability $\left[\mathrm{h}^{2}=\right.$ $\left.4 \sigma_{\mathrm{s}}^{2} /\left(\sigma_{\mathrm{h}}^{2}+\sigma_{\mathrm{s}}^{2}+\sigma_{\mathrm{e}}^{2}\right)\right]$ of liability to sucking deficiency in a multiple-trait liability analysis (SA6 $=$ sucking ability at first meal; SA12 = sucking ability at second meal; SA24 = sucking ability at third meal; SA48 = sucking ability on second day; SA72 = sucking ability on third day).

cent meals. In Figure 5, rerankings of the sires in the multiple-trait analysis are reported. Reranking was calculated as the difference in rank between the breeding values of a sire obtained at different meals (e.g., rank of sire EBV at 6 and $12 \mathrm{~h}$ ). As expected, reranking was considerably less for adjacent measures of sucking behavior. In addition, reranking for early meals ( 6 and $12 \mathrm{~h}$ ) was smaller than reranking for subsequent observations.

What is measured here is both the innate ability of the calf to know how to suck and the willingness of the calf to express that behavior, which may be related to myriad influences in the neonatal period, some of which could be genetically influenced. Even if here the 2 as-

Table 3. Heritability ${ }^{1}$ (diagonal), genetic correlations (above), and phenotypic correlations (below) for each of the 5 meals in the multivariate analysis ${ }^{2}$

\begin{tabular}{llllll}
\hline Item & \multicolumn{1}{c}{ SA6 } & \multicolumn{1}{c}{ SA12 } & \multicolumn{1}{c}{ SA24 } & \multicolumn{1}{c}{ SA48 } & SA72 \\
\hline SA6 & $0.26( \pm 0.09)$ & 0.97 & 0.85 & 0.82 & 0.84 \\
SA12 & 0.94 & $0.23( \pm 0.08)$ & 0.87 & 0.91 & 0.91 \\
SA24 & 0.82 & 0.97 & $0.22( \pm 0.09)$ & 0.92 & 0.94 \\
SA48 & 0.69 & 0.79 & 0.81 & $0.13( \pm 0.08)$ & 0.99 \\
SA72 & 0.57 & 0.63 & 0.64 & 0.91 & $0.13( \pm 0.07)$ \\
\hline
\end{tabular}

${ }^{1}$ Heritability: $\mathrm{h}^{2}=4 \sigma_{\mathrm{s}}^{2} /\left(\sigma_{\mathrm{s}}^{2}+\sigma_{\mathrm{h}}^{2}+\sigma_{\mathrm{e}}^{2}\right)$, where $\sigma_{\mathrm{s}}^{2}=$ sire variance, $\sigma_{\mathrm{h}}^{2}=$ herd variance, and $\sigma_{\mathrm{e}}^{2}=$ residual variance.

${ }^{2}$ SA6 = sucking ability at first meal; SA12 = sucking ability at second meal; SA24 = sucking ability at third meal; SA48 = sucking ability on second day; SA72 = sucking ability on third day. 

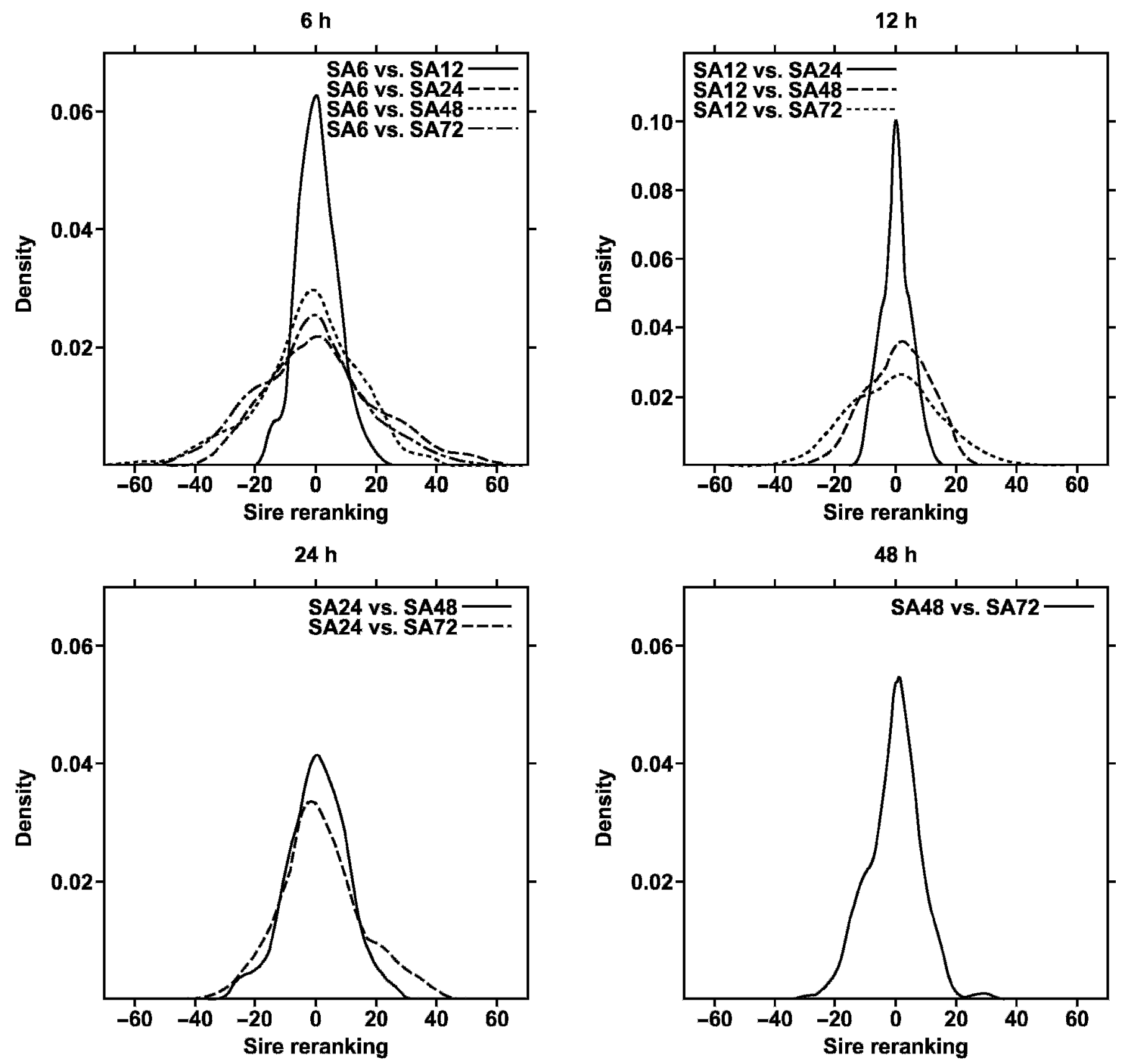

Figure 5. Distribution of the change in sire ranking between evaluations at different meals $(\mathrm{SA} 6=$ sucking ability at first meal; SA12 = sucking ability at second meal; SA24 = sucking ability at third meal; SA48 = sucking ability on second day; SA72= sucking ability on third day).

pects may not be discerned, given the structure of data collection, the intent was to address the basis of the calf's willingness suck and how quickly the calf learned to do it.

Variance component estimates indicated that the genetic component of sucking ability was large enough to be considered as a possible selection criterion. In the single-trait analysis, the heritability value is 0.14 . Be- cause all the observations for each calf were recorded within $3 \mathrm{~d}$, the temporary environmental component could play an important role in increasing the variability of traits. Indeed, many changes occur during the first days of life, because calves that lack a strong sucking ability in an earlier meal usually learn to consume feed from a nipple pail or bottle. The ability to learn is also strongly related to the farmers' help in the first or 
second day of life, which can be considered a part of the temporary environmental variance. Heritability estimates in the multiple-trait analysis decreased substantially from SA6 to SA72. These results suggest that the variation in the innate ability to suck is more easily detectable in the early stages of life. Consequently, in recording a trait to be used in a selection program, sucking ability should be recorded within the first 12 $\mathrm{h}$ of life for each individual. Estimated genetic correlations among traits were large, indicating that measurements of sucking ability at different intervals from birth were essentially the same trait. In the future, data from a wider range of herd environments and a greater proportion of the population should be collected, and researchers should investigate maternal components of these traits.

\section{CONCLUSIONS}

Results of this study showed that SA6 and SA12 have moderate heritability ( 0.26 and 0.23$)$ and can be identified as traits to use in a selection scheme. According to these results, selection to increase sucking ability is possible, but a larger test group will be needed to ensure sufficient accuracy of sire EBV predictions.

For future data collection, measurements on the first day after birth should be used. In the Italian recording system, the information on sucking ability is easily recorded when calves receive their ear tags. Additional studies are necessary to confirm these findings and to collect additional information to determine whether to incorporate sucking ability in a national selection program.

\section{REFERENCES}

Appleby, M. C., and D. M. Weary. 2001. Beverley Chua performance and feeding behavior of calves on ad libitum milk from artificial teats. Appl. Anim. Behav. Sci. 74:191-201.

Blass, E. M. 2003. Mammary messages. Nature 424:25-26.

Blum, J. W., and H. Hammon. 2000. Colostrum effect on gastrointestinal tract, and on nutritional, endocrine and metabolic parameters in neonatal calves Livest. Prod. Sci. 66:151-159.

Bulot, O. 2004. Strong and weak points of the brown breed compared to other dairy breeds: How to maximise production in a competitive environment. Pages 171-177 in Proc. 7th World Conf. Brown Swiss Cattle Breeders. Publ. ANARB, Bussolengo, Italy.

Bush, L. J., and T. E. Staley. 1980. Absorption of colostral immunoglobulins in newborn calves. J. Dairy Sci. 63:672-680.

Erf, D. F., L. B. Hansen, and R. R. Neitzel. 1990. Inheritance of calf mortality for brown Swiss cattle. J. Dairy Sci. 73:1130-1134.

Faber, S. N., N. E. Faber, T. C. McCauley, and R. L. Ax. 2005. Effects of colostrum ingestion on lactational performance. Prof. Anim. Sci. 21:420-425.
Friend, T. H., G. R. Dellmeier, and E. E. Gbur. 1985. Comparison of four methods of calf confinement. I. Physiology. J. Anim. Sci. 60:1095-1101.

Friend, T. H., L. Taylor, G. R. Dellmeier, D. A. Knabe, and L. A. Smith. 1988. Effect of confinement method on physiology and production of gestating gilts. J. Anim. Sci. 66:2906-2915.

Gianola, D. 1982. Theory and analysis of threshold characters. J. Anim. Sci. 54:1079-1096.

Hansen, M., P. Madsen, J. Jensen, J. Pedersen, and L. G. Christensent. 2003. Genetic parameters of postnatal mortality in Danish Holstein Calves. J. Dairy Sci. 86:1807-1817.

Harbers, A., L. Segeren, and G. de Jong. 2000. Genetic parameters for still birth in Netherlands. Interbull Bull. 25:1-6.

Heringstad, B., D. Gianola, Y. M. Chang, J. Ødegård, and G. Klemetsdal. 2006. Genetic associations between clinical mastitis and somatic cell score in early first-lactation cows. J. Dairy Sci. 89:2236-2244.

Johanson, J. M., and P. J. Berger. 2003. Birth weight as a prediction of calving ease and perinatal mortality in Holstein cattle. J. Dairy Sci. 86:3745-3755.

Lay, D. C., Jr., T. H. Friend, K. K. Grissom, R. L. Hale, and C. L. Bowers. 1992. Novel breeding box has variable effects on heart rate and cortisol response of cattle. Appl. Anim. Behav. Sci. 35:1-10.

Lay, D. C., Jr., R. D. Randel, T. H. Friend, O. C. Jenkings, D. A. Neuendorff, D. M. Bushong, E. K. Lanier, and M. K. Bjorge. 1997. Effects of prenatal stress on suckling calves. J. Anim. Sci. 75:3143-3151.

Mitchell, G., J. Hattingh, and M. Ganhao. 1988. Stress in cattle assessed after handling, after transport and after slaughter. Vet. Rec. 123:201-205.

Nowak, R., T. M. Murphy, D. R. Lindsay, P. Alster, R. Andersson, and K. Uvnas-Moberg. 1997. Develop of preferential relationship with the mother by the newborn lamb: Importance of sucking activity. Physiol. Behav. 62:681-688.

de Passilè, A. M. 2001. Suckling motivation and related problems in calves. Appl. Anim. Behav. Sci. 72:175-178.

Santus, E. 2004. The Brown breed in Italy and ANARB: A winning combination. Pages 15-23 in Proc. 7th World Conf. Brown Swiss Cattle Breeders. Publ. ANARB, Bussolengo, Italy.

Schaal, B., G. Coureaud, D. Langlois, C. Giniès, E. Sémon, and G. Perrier. 2003. Chemical and behavioural characterization of the rabbit mammary pheromone. Nature 424:68-72.

Snell, E. J. 1964. A scaling procedure for ordered categorical data. Biometrics 20:592-607.

Sorensen, D., and D. Gianola. 2002. Likelihood, Bayesian, and MCMC Methods in Quantitative Genetics. Springer-Verlag, New York, NY.

Staley, T. E., and L. J. Bush. 1985. Receptor mechanisms of the neonatal intestine and their relationship to immunoglobulin absorption and disease. J. Dairy Sci. 68:184-205.

Steinbock, L., A. Nasholm, B. Berglunb, K. Johanssons, and J. Philipsson. 2003. Genetic effects on stillbirth and calving difficulty in Swedish Holstein at first and second calving. J. Dairy Sci. 86:2228-2235.

Stott, G. H., D. B. Marx, B. E. Menefee, and G. T. Nightengale. 1979. Colostral immunoglobulin transfer in calves I. Period of absorption. J. Dairy Sci. 62:1632-1638.

Wittum, T. E., and L. J. Perino. 1995. Passive immune status at postpartum hour 24 and long-term health and performance of calves. Am. J. Vet. Res. 56:1149-1154.

Zemp, M. 2004. Brown Swiss cattle breeding in Western Europe. Pages 25-31 in Proc. 7th World Conf. Brown Swiss Cattle Breeders. Publ. ANARB, Bussolengo, Italy. 